\title{
Verticilliose de la tomate : conséquences des inter- actions entre l'agressivité de l'agent pathogène et la résistance de l'hôte au niveau des populations
}

Issa BEYE \& Jean-François LAFAY (1)

Laboratoire de Cryptogamie associé au C.N.R.S. (UA 086), Université Paris-Sud, Bâtiment 400, F91405 Orsay Cedex

RÉSUMÉ

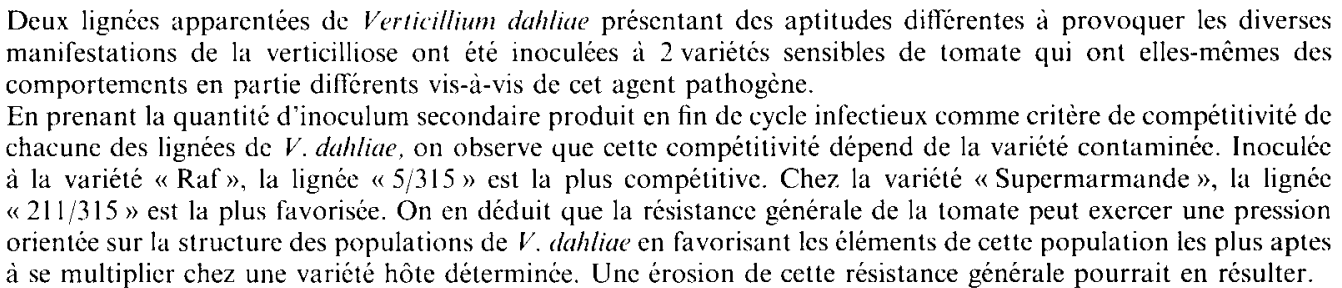

Mots clés additionnels : Résistancé générale, Verticillium dahliae, lignées, variétés, compétitivité.

Verticillium wilt of tomato: consequences of interactions between pathogen aggressiveness and resistance of the host at the population level.

Two Vericillium duhliae strains exhibiting different aptitudes in inducing the various manifestations of Verticillium hadromycosis were inoculated to two susceptible tomato varieties which themselves exhibit different behaviours towards the pathogen. The amount of secondary inoculum produced at the end of the infection cycle, taken as the competitivity critcrion of a $V$. dahliae strain, showed that this competitivity depended on the variety inoculated. When inoculated to $\mathrm{cv}$. 'Raf, strain ' $5 / 315^{\prime}$ was the most competitive, whereas inoculation to $\mathrm{cv}$. 'Supermarmande' favoured strain ' $211 / 315$ '. These results show that the general resistance of tomato may exert a selection pressure on the population structure of $V$. dahliae by favouring, within such populations, the strains which are better adapted to multiply in a certain host variety. A decrease in this general resistance may result.

Additional key words : General resistance, Verticillium dahliae, strains, varieties, competitivity.

\section{INTRODUCTION}

Résistance verticale et résistance horizontale sont 2 termes pratiques introduits par VAN DER PLANK (1968) pour désigner 2 types de manifestations de la résistance des plantes aux maladies. Les effets sélectifs de la résistance verticale sur la structure d'une population pathogène sont bien connus: ils favorisent la prédominance des races capables de surmonter le gène concerné. En revanche, l'existence de pressions sélectives exercées par la résistance générale sur la structure

(1) Toute corespondance est à adresser à J. F. LAFAY (Orsay) des populations pathogènes demeure un sujet de controverse.

Dans le cas du couple formé par la tomate et Verticillium dahliae Kleb., BEYE \& LAFAY (1988) ont montré qu'il peut exister des interactions souches $\times$ variétés dans des relations hôte-parasite qui ne mettent en jeu que l'agressivité de l'agent pathogène et la résistance générale de l'hôte. En prenant en compte plusieurs manifestations de la verticilliose, ils ont montré que, dans certaines confrontations, une souche de Verticillium dahliae très agressive à en juger par l'une des expressions de la maladie chez la plante l'est beaucoup moins si l'on en juge par une autre. De la même façon, ils ont montré qu'un cultivar classé comme possédant 
un bon niveau de résistance générale d'après un critère peut être classé comme médiocre selon un autre critère.

L'observation de telles interactions entre certaines au moins des composantes de l'agressivité et des composantes de la résistance générale soulève par analogie avec les interactions entre virulence et résistance spécifique, une question au niveau des populations: la résistance générale peut-elle exercer sur une population pathogène agressive, une pression orientée à l'avantage des éléments de cette population les mieux armés pour surmonter une ou des composantes déterminées de cette résistance générale?

Répondre à cette question dans le cas d'une affection localisée comme le sont beaucoup d'affections foliaires, ne présente pas de difficultés, surtout si le cycle infectieux est court par rapport au cycle de développement de l'hôte. Il est alors aisé de mettre en compétition des souches pathogènes présentant des caractéristiques différentes et de suivre au fil des cycles infectieux successifs les changements survenus dans la proportion des différents génotypes: les plus compétitifs doivent devenir plus ou moins tôt les éléments prédominants. C'est par une telle méthode que Chevaugeon er al. (1978) ont déterminé chez Pyricularia oryzae la perte de compétitivité liée à la possession d'allèles de virulence vis-à-vis des gènes de résistance $P i-t a$ et $P i-t a^{2}$ et par conséquent la force de ces gènes de résistance. Mais un tel protocole est mal adapté lorsqu'il s'agit d'une maladie vasculaire qui se généralise à toute la plante et dont l'agent ne se dissémine de plante à plante que très tardivement. Même s'il était concevable de mettre simultanément en compétition plusieurs souches de $V$. dahliae chez le même individu-hôte, il serait impossible d'estimer la part qui revient à chaque souche dans la quantité de maladie mesurée.

Nous avons cherché à contourner cette difficulté en considérant qu'au fil des générations de $V$. dahliae, c'est-à-dire au fil des infections successives par l'intermédiaire du sol, c'est l'abondance relative des microsclérotes formés sur une plante qui détermine la probabilité pour chaque génotype pathogène d'infecter une autre plante. En conséquence, nous avons considéré que des différences dans la production de microsclérotes chez une variété de tomate reflèteraient d'éventuelles différences de compétitivité entre génotypes de $V$. dahliae vis-à-vis de cette variété. Cette hypothèse est confortée par les travaux d'autres auteurs. Selon Wilhelm (1955), dans un sol infesté par Verticillium, l'évolution du potentiel infectieux dépend la plupart du temps des rotations culturales : il augmente rapidement en présence d'hôtes sensibles comme la tomate, la pomme de terre ou le cotonnier; mais il ne dépend pas de la vie saprophytique du champignon dans le sol. Bouнot (1980) souligne les conséquences de la biologie des champignons telluriques sur les variations de l'inoculum. Il distingue 2 catégories de parasites: d'un côté les champignons comme les Pythium Pringsh. pour lesquels le potentiel infectieux du sol dépend plus des conditions écologiques et de leurs effets sur les activités saprophytiques de ces champignons que de la densité d'inoculum initiale, et d'un autre côté les « root inhabiting fungi ", tels que les Fusarium oxysporum (Schlecht.) Snyd. \& Hans., Verticillium Nees ex Lk. et Ophiobolus Riess dont la multiplication, et par conséquent la production d'inoculum, s'effectue essentiellement aux dépens de l'hôte. De même, pour Evans et al. (1966), la croissance saprophytique de $V$. dahliac dans le sol ne contribue pas à l'augmentation de l'inoculum; seule la plante-hôte, le cotonnier en l'occurence, fournit un support pour sa multiplication.

C"est sur ces considérations que nous nous sommes appuyés pour comparer la compétitivité de 2 lignées de $V$. dahliae qui sont toutes deux agressives à l'égard de la tomate mais qui présentent des aptitudes différentes à provoquer les diverses manifestations de la verticilliose (BEYE \& LAFAY, 1988). Ces 2 lignées ont été inoculées à 2 variétés sensibles de tomate qui présentent ellesmêmes des différences quantitatives dans leurs réponses à l'agent pathogène.

\section{MATÉRIEL ET MÉTHODES}

\section{A. L'hôte et l'agent pathogène}

Les 2 variétés de tomate «Supermarmande» et «Raf» sont utilisées. Les semis, l'obtention des plantes et les conditions de serre sont décrits par BEYE \& LAFAY (1985). Les 2 lignées de $V$. dahliae « 5/315» et «211/315» ont été obtenues par SEBTI (1982). Les conditions de culture et de conservation des lignées, la production de l'inoculum, les procédés de contamination des plantes ont également été décrits par BEYE \& LAFAY (1985).

\section{B. Estimation de l'inoculum secondaire}

\section{Préparation des plantes}

Trente-cinq plantes ont été utilisées pour chacun des 4 couples qui peuvent être formés avec l'une ou l'autre des 2 lignées de $V$. dahliae et l'une ou l'autre des 2 variétés de tomate. Les plantes appartenant à un même couple sont récoltées 6 semaines après la fin de la période d'incubation. Chaque plante entière est enveloppée individuellement dans un papier filtre non stérile et déposée dans un récipient exposé à l'air libre de la serre.

Six semaines plus tard, chaque plante est transférée dans un sachet en polyéthylène, mise au congélateur à $20{ }^{\circ} \mathrm{C}$ pendant $24 \mathrm{~h}$ puis lyophilisée. La congélation et la lyophilisation, d'une part, tuent les constituants autres que les microsclérotes et, d'autre part, améliorent l'efficacité des opérations ultérieures d'extraction.

Le matériel sec est ensuite réduit en poudre par des broyages successifs au mortier dans l'azote liquide suivis de tamisages sur tamis de $1 \mathrm{~mm}$ de maille. Plante par plante, la poudre est pesée et conservée au sec à la température du laboratoire dans un récipient de verre, en vue du dénombrement des microsclérotes.

\section{Dénombrement des microsclérotes}

Plusieurs techniques ont été proposées aussi bien pour dénombrer les microsclérotes, dans le sol ou dans la plante, que pour déterminer leur viabilité. 
Ashworth et al. (1972), Menzies \& Griebel (1967), NADAVAKuren \& Horner (1959) ont proposé des procédés de mesure qui font appel à des milieux de culture plus ou moins sélectifs avec ou sans dilution préalable de l'échantillon de sol à analyser.

TSAI \& ERWIN (1975) ont utilisé la colorimétrie pour évaluer la quantité de microsclérotes contenue dans un homogénat de milieu de culture. IOANNOU et al. (1977) préparent des segments de tige de tomate de $2 \mathrm{~cm}$ de long, les broient dans $20 \mathrm{ml}$ d'eau distillée et comptent à la cellule à nématodes, les microsclérotes contenus dans $1 \mathrm{ml}$ de broyat. Enfin, Evans et al. (1966) enlèvent l'écorce des tiges de cotonnier et estiment par microphotographie le nombre de sclérotes présents dans la moelle.

Pour notre part, après des essais comparatifs, nous avons retenu le protocole suivant. Le broyat pulvérulent est incorporé à un milieu nutritif gélosé maintenu en surfusion. Ce milieu est un bouillon de pomme de terre glucosé et gélosé (P.D.A.), auquel sont ajoutés 100 p.p.m. de streptomycine et 100 p.p.m. de pénicilline $\mathrm{G}$ après autoclavage et juste avant utilisation. Le taux de dilution choisi $1 / 1000(\mathrm{P} / \mathrm{V})$ permet d'obtenir des nombres de sclérotes par boîte de Petri qui peuvent être recensés. Cette dilution est obtenue en 2 temps : $1 \mathrm{~g}$ de poudre végétale est diluée dans $9 \mathrm{ml}$ d'eau stérile puis, après agitation, $1 \mathrm{ml}$ de cette première suspension contenant donc $100 \mathrm{mg}$ de broyat par $\mathrm{ml}$ est dispersé dans $99 \mathrm{ml}$ de milieu de culture maintenu dans un bainmarie à $55^{\circ} \mathrm{C}$. L'ensemble est distribué dans 5 boîtes de Petri, à raison de $20 \mathrm{ml}$ par boîte. Les thalles qui naissent de la germination des microsclérotes sont comptés après $48 \mathrm{~h}$ de culture à $26^{\circ} \mathrm{C}$ et à l'obscurité. L'analyse statistique des résultats a été effectuée au moyen du logiciel S.P.S.S. (Statistical Package for the Social Sciences) implanté à l'Univac 1110 du Centre Paris-Sud Informatique.

\section{RÉSULTATS}

A l'aide du test de Kolmogorov et Smirnov (d'après FERIGNAC, 1962) nous avons vérifié que pour toutes les plantes, les nombres de thalles observés dans les 5 boîtes ensemencées avec le broyat d'une même plante se distribuaient suivant une loi de Poisson, c'est-à-dire au hasard. La distribution de Poisson s'est révelée acceptable dans tous les cas (risque $<5$ p. 100). Par ailleurs. quelle que soit la combinaison variété $\times$ lignée, le poids sec moyen des plantes ne varie pas significativement et est toujours voisin de 2,6 grammes. Les résultats obtenus pour $100 \mathrm{mg}$ de poudre sont donc directement utilisables dans les calculs, sans qu'il soit nécessaire de les ramener au poids de la plante entière.

L'analyse de variance à un critère de classification (la combinaison variété $\times$ lignée) montre (tabl. 1a) qu'il y a des différences significatives entre les 2 lignées lorsqu'elles sont inoculées à l'une ou à l'autre variété, ainsi qu'entre les 2 variétés selon qu'elles sont infectées par l'une ou l'autre lignée. L'analyse de variance à 2 dimensions (tabl. 1b) montre que l'effet lignée et l'effet variété ne sont pas significatifs individuellement mais que c'est l'interaction lignée $\times$ variété qui est hautement significative. Ceci traduit le fait que les quantités de microsclérotes produites par chacune des lignées sur la variété qui lui est favorable $(74,5 \pm 3,5$ et $75,1 \pm 3,5)$ ne sont pas statistiquement différentes, de même que les productions de chacune des lignées sur la variété qui lui est défavorable $(44,7 \pm 2,0$ et $44,8 \pm 2,2)$. Le test de DUNCAN (1955) (tabl.2) montre que sur la variété "Raf » la lignée « $5 / 315$ » produit un inoculum secondaire beaucoup plus abondant que la lignée « $211 / 315$ ». Sur la variété "Supermarmande», ce classement est inversé : la lignée «211/315» est significativement la plus productive.

\section{DISCUSSION ET CONCLUSION}

Si l'on fait l'hypothèse qu'après plusieurs cycles culturaux successifs, la lignee prédominante sur une variété donnée sera celle qui produit le plus d'inoculum secondaire viable à chaque cycle infectieux, nous pouvons prévoir que la lignée qui prédominera sur la variété "Raf» sera la lignée « $5 / 315$ » et que sur la variété "Supermarmande», elle cédera la première place à la lignée «211/315». Cela montre que la résistance générale n'est pas neutre. Elle fait subir aux génotypes

TABLF:AU

Analyes de variance des nombres de colonies par $100 \mathrm{mg}$ de phante.

Analyses of lariance of the mmmer of colomices per $100 \mathrm{mg}$ of plam.

\begin{tabular}{|c|c|c|c|c|c|}
\hline $\begin{array}{l}\text { Source } \\
\text { de variation }\end{array}$ & $\begin{array}{c}\text { Degrés } \\
\text { de liberté }\end{array}$ & $\begin{array}{l}\text { Somme des } \\
\text { carrés } \\
\text { des ecarts }\end{array}$ & $\begin{array}{l}\text { Carré } \\
\text { moyen }\end{array}$ & $\mathrm{F}$ & $\begin{array}{c}\text { Probabilite } \\
\text { de F }\end{array}$ \\
\hline \multicolumn{6}{|l|}{$-1 a$} \\
\hline Entre les 4 combinaisons & 3 & 31595.79 & 10531,93 & 149,79 & $<0,001$ \\
\hline Résiduelle & 136 & 9568,34 & 70,31 & & \\
\hline $\begin{array}{l}-1 \mathrm{~b} \\
\text { Effet lignée }\end{array}$ & 1 & 0.55 & 0.55 & 0,01 & 0,93 \\
\hline Effet variété & 1 & 0,83 & 0,83 & 0,01 & 0,91 \\
\hline Interact. lignée $\times$ variété & 1 & 31515.81 & 31515,81 & 446,18 & $<0,001$ \\
\hline Résiduelle & 135 & 9353,71 & 70.63 & & \\
\hline
\end{tabular}

Combinaisons lignée variété (voir tabl. 2).

The strain $\times$ variety combinations are in table 2 . 
TABLEAU 2

Nombre moyen de colonies pour $100 \mathrm{mg}$ de plante par combinaison lignée $\times$ variété

et test de rang de DUCAN.

DUNCAN range test of the average number of colonies per $100 \mathrm{mg}$ of plant

for each strain $\times$ variety combination

\begin{tabular}{ccc}
\hline \multirow{2}{*}{ Lignée } & \multicolumn{2}{c}{ Variété } \\
\cline { 2 - 3 } & Raf & Supermarmande \\
\cline { 2 - 3 } $5 / 315$ & $\mathrm{~A}$ & $\mathrm{~B}$ \\
& $74,5 \pm 3,5$ & $44,8 \pm 2,2$ \\
\hline $211 / 315$ & $\mathrm{~B}$ & $\mathrm{~A}$ \\
& $44,7 \pm 2,0$ & $75,1 \pm 3,5$ \\
\hline
\end{tabular}

* Les intervalles de confiance sont au seuil de 5 p. 100.

* $5 \%$ confidence interval.

du parasite des pressions de sélection qui favorisent le plus apte à contourner l'ensemble des différents obstacles que lui opposent chacune de ses composantes. Pour tenter de déterminer quelles sont les composantes concernées, on peut se reporter à une autre étude (BEYE \& LAFAY, 1988) dont les résultats ont précisément orienté notre choix des 2 lignées et des 2 variétés utilisées ici, et où nous avons comparé, entre autres, les différentes expressions de l'agressivité des lignées « $5 / 315$ » et « $211 / 315$ » et de la résistance des variétés "Raf» et « Supermarmande ». L'apparition de la maladie intervient après un délai un peu plus court chez la variété "Raf» contaminée avec la lignée «5/315» qu'avec la lignée « $211 / 315$ ». De même, chez la variété "Raf », la lignée « $5 / 315$ » induit des altérations foliaires plus graves et s'étendant plus rapidement; elle provoque un retard de croissance plus marqué et elle freine davantage l'émission des feuilles. Chez la variété «Supermarmande", les 2 lignées de $V$. dahliae font apparaître des symptômes foliaires après des temps d'incubation statistiquement identiques, mais les altérations foliaires sont plus marquées et surtout la vitesse d'extension de ces altérations est plus élevée après inoculation de la lignée $~\langle 5 / 315 »$; en revanche, la diminution de la taille des plantes et le ralentissement de l'émission des feuilles sont beaucoup plus accusés à la suite de l'inoculation de la lignée «211/315».

En rapprochant les résultats de ces 2 études, on peut penser que, dans le cas présent, les éléments discriminants responsables des interactions entre lignées et variétés seraient, chez le parasite les facteurs d'agressivité qui se traduisent par des actions sur la croissance de l'hôte, et chez l'hôte les facteurs de résistance générale qui s'opposent à ces actions.

En résumé, nous décrivons une situation où, dans des relations hôte-parasite basées sur des formes générales de la résistance et des formes agressives du pouvoir pathogène, les interactions hôte-parasite peuvent impliquer des composantes partielles de l'agressivité et de la résistance générale. En fonction des composantes en présence, certains éléments de la population pathogène peuvent être mieux armés face à une variété donnée, lui infligeant des dégâts plus importants et produisant davantage d'inoculum secondaire, lequel inoculum, issu d'une lignée plus agressive, sera potentiellement plus dangereux lors d'un prochain cycle cultural de la même variété.

Ainsi la présente étude, bien que menée sur un nombre limité de couples variété $\times$ lignée, montre qu'une population parasite peut contourner le dispositif de résistance générale d'une variété en renforçant telle ou telle facette de l'agressivité. Effectivement, l'érosion de la résistance générale sous l'effet de variations de l'agressivité de la population pathogène a déjà été observée et discutée. Cross (1963) cité par VAN DeR Plank (1978) dans le cas du couple Xanthomonas malvacearum - cotonnier, plus près de nous, SKAJENNIKOFF \& RAPILLY (1983) (Septoria nodorum Berk. blé), Ashworth et al. (1983) (Verticillium dahliae coton) avaient signalé ce danger.

Pourtant, il existe des arguments génétiques et physiologiques en faveur de la stabilité de la résistance générale. A long terme, l'homéostase génétique favorise les intermédiaires et non les extrêmes. Il y aurait sélection stabilisatrice en faveur des éléments moyennement agressifs de la population pathogène, les extrêmes étant désavantageux (VAN DER PlanK, 1968). Par ailleurs, on peut penser que la plupart des activités physiologiques impliquées dans l'agressivité participent simultanément à d'autres fonctions indispensables à la vie de l'agent pathogène. En conséquence toute modification de l'équilibre entre ces nombreuses activités dans le sens d'une agressivité accrue peut avoir un effet dépressif sur les autres fonctions et réduire la compétitivité. Ce sont probablement des arguments de ce type, conjugués au fait que la résistance générale, procédant de facteurs multiples, présente des variations quantitatives et continues, qui ont conforté l'idée de la stabilité apparente de cette résistance générale.

En conclusion, l'utilisation de la résistance générale dans la sélection des variétés présente un risque analogue à celui inhérent à la résistance spécifique. Est-il alors possible de se prémunir contre la possible diminution d'efficacité de la résistance générale en s'inspirant des stratégies utilisant la résistance spécifique, telles que l'utilisation de mélanges de variétés à composantes de résistance complémentaires, ou de variétés où l'on aurait accumulé les composantes de résistance les plus favorables?

Reçu le 21 mai 1987. Accepté le 23 février 1988.

\section{REMERCIEMENTS}

Les auteurs remercient Monsieur le Professeur Chevaugeon pour ses nombreux avis et conseils dans la préparation et la réalisation du manuscrit. Ils remercient également Monsieur Guenard des Ets Clause pour la fourniture des semences, ainsi que Monsieur J. C. Bousson pour le transfert des données au Centre de Calcul. 


\section{RÉFÉRENCES BIBLIOGRAPHIQUES}

Ashworth L. J., Waters J. E., George A. G., McCutcheon O. D., 1972. Assessment of microsclerotia of Verticillium albo-atrum in fields soils Phytopathology, 62, 715-719.

Ashworth L. J. Jr., Galanopoulos N., Galanopoulos S., 1983. Selection of pathogenic strains of Verticillium dahliae and their influence on the useful life of cotton cultivars in the field. Phvtopathology, 73, 1637. 1639.

Beye I., Lafay J. F., 1985. Etude de critères de sélection pour une résistance générale à la verticilliose de la tomatc. Agronomie, 5, 305-311.

Beye I., Lafay J. F., 1988. Verticilliose de la tomate: Analyse des interactions entre l'agressivité de l'agent pathogène et la résistance générale de l'hôte. Agronomie, 8 (4), 351-361.

Bouhot D., 1980. Le porentiel infectieux des sols. Un concept, un modile pour sa mesure, quelques applications. Thèse Doctorat ès Sciences. Univ. Nancy I, p. 11-12.

Chevaugeon J., Bah C. P., Makounzi J. A., 1978. Mesure rapide de la force des gènes de résistance. Application à la pyriculariose du riz. Ann. Phytopathol., 10, 277-286.

Duncan D. B., 1955. Multiple range and multiple F-test. Biometrics, 11, 1-42.

Evans G., Snyder W. C., Wilhelm S., 1966. Inoculum increase of the Verticillium wilt fungus in cotton. Phytopathology, 56, 590-594

Ferignac P., 1962. Test de Kolmogorov-Smirnov sur la validité d'une fonction de distribution. Rev. Statist. appliquée, 10, 13-32.
Ioannou N., Schneider R. W., Grogan R. G., 1977. Effect of oxygen, carbon dioxide and ethylene on growth, sporulation, and production of microsclerotia by Verticillium dahliae. Phytopathology, 67, 645-650

Menzies J. D., Griebel G. E., 1967. Survival and saprophytic growth of Verticillitum dahliae in uncropped soil. Phytopathology, 57, 703-709.

Nadakavukaren M. J., Horner C. E., 1959. An alcohol agar medium selective for determining Verticillium microsclerotia in soil. Phytopathology, 49, 527-528.

Sebti S., 1982. Essai d'analyse des composantes du pouvoir pathogène de Verticillium albo-atrum Reinke et Berthold. Thèse Doctorat $3^{\mathrm{e}}$ Cycle. Univ. Paris-Sud, Orsay, 93 p.

Skajennikoff M., Rapilly F., 1983. Etudes sur l'agressivité de Septoria nodorum Berk.: Effet des hôtes (tricicale et Blé) et des organes attaqués. Agronomie, 3, (2) 131-140.

Tsai S. D., Erwin D. C., 1975. A method of quantifying numbers of microsclerotia of Verticillium albo-atrum in cotton plant tissue and in pure culture. Phytopathology, 65, 1027-1028.

Van Der Plank J. E., 1968. Disease resistance in plants. Acad. Press. Inc. New York, London, $206 \mathrm{p}$

Van Der Plank J. E., 1978. Genetic and molecular basis of plant pathogenesis. Springer-Verlag, Berlin, Heidelberg, New York, 168 p.

Wilhelm S., 1955. Longevity of the Verticillium wilt fungus in the laboratory and field. Phytopathology, 45, 180-181. 